\title{
Publisher's Note: Laser Theory for Optomechanics: Limit Cycles in the Quantum Regime [Phys. Rev. X 4, 011015 (2014)]
}

Niels Lorch, Jiang Qian, Aashish Clerk, Florian Marquardt, and Klemens Hammerer (Received 7 February 2014; published 20 February 2014)

DOI: 10.1103/PhysRevX.4.019902

Subject Areas: Nanophysics, Quantum Physics

This paper was published online on 31 January 2014 with incomplete references. Reference [28] should read as "P. D. Nation, Nonclassical Mechanical States in an Optomechanical Micromaser Analogue, Phys. Rev. A 88, 053828 (2013)"; Ref. [44] should read as "S. Aldana, C. Bruder, and A. Nunnenkamp, On the Equivalence between an Optomechanical System and a Kerr Medium, Phys. Rev. A 88, 043826 (2013)"; Ref. [49] should read as "S. Kilin and A. Mikhalychev, Single-Atom Laser Generates Nonlinear Coherent States, Phys. Rev. A 85, 063817 (2012).” The references have been corrected as of 11 February 2014. 